\title{
BELTRAMI EQUATION AND CLUSTER LENSING
}

\author{
Characteristic Equations \& Applications
}

\author{
T. SCHRAMM \\ Technical University of Hamburg-Harburg \\ Computer Center - D 21071 Hamburg \\ and \\ Hamburg Observatory \\ Gojenbergsweg 112 - D 21029 Hamburg
}

\begin{abstract}
Arclets in clusters of galaxies can be used to determine the lens mapping and not only to constrain the mass density of the cluster. Multiply imaged arclets are therefore easily identified without further modeling.
\end{abstract}

\section{The Beltrami equation}

In Schramm \& Kayser (1995) we introduced the complex Beltrami Equation as an appropriate framework for the analysis of arclets in cluster lensing. Corresponding real formalisms have been developed by Kaiser and Schneider \& Seitz (this volume, compare also the references in Schramm \& Kayser 1995). Here, we show how the solutions of the Beltrami differential equation can be used to identify multiply imaged arclets. The Beltrami Equation

$$
\frac{\partial w}{\partial \bar{z}}=\mu \frac{\partial w}{\partial z}
$$

states that a small ellipse in the deflector $(z=x+\mathrm{i} y)$ plane given by $\mu$ is mapped locally by $w$ onto a circle in the source $(w=u+\mathrm{i} v)$ plane. The axial ratio $\epsilon$ of the ellipse and the direction angle $\varphi$ are given by $\epsilon=(1-|\mu|) /(1+|\mu|)$ and $2 \varphi=\pi+\arg (\mu)$, respectively.

\section{Known source sizes}

The Jacobian is also easily found

$$
J=\left|\frac{\partial w}{\partial z}\right|^{2}-\left|\frac{\partial w}{\partial \bar{z}}\right|^{2}
$$


Trivially, the mass density $\sigma$ is uniquely determined if the Jacobian and the Beltrami parameter can be measured at the location of an arclet. Since $\partial w / \partial z=1-\sigma$ we can solve the Beltrami Equation and the Jacobian for the mass density:

$$
(1-\sigma)^{2}=\frac{J}{1-|\mu|^{2}}
$$

\section{Characteristic equations}

Normally we are not so lucky to have the Jacobian but we assume we can measure the $\mu$-field with some accuracy. However, the formulation as differential equation yields some insights. For the measurable Beltrami parameter we find for (one-plane) lens mappings

$$
\mu=\mu_{r}+\mathrm{i} \mu_{i}=\frac{\frac{\partial u}{\partial x}-\frac{\partial v}{\partial y}+2 \mathrm{i} \frac{\partial v}{\partial x}}{\frac{\partial u}{\partial x}+\frac{\partial v}{\partial y}}
$$

which results in two decoupled linear, homogeneous partial differential equations

$$
\mu_{i} \frac{\partial u}{\partial x}-\left(1+\mu_{r}\right) \frac{\partial u}{\partial y}=0 \quad,\left(1-\mu_{r}\right) \frac{\partial v}{\partial x}-\mu_{i} \frac{\partial v}{\partial y}=0
$$

The characteristics of these equations are given by:

$$
\begin{aligned}
& x^{\prime}(t)=\mu_{i}, \quad y^{\prime}(t)=-\left(1+\mu_{r}\right) \Longrightarrow \frac{\mathrm{d} y}{\mathrm{~d} x}=-\frac{1+\mu_{r}}{\mu_{i}} \\
& x^{\prime}(t)=\left(1-\mu_{r}\right), \quad y^{\prime}(t)=-\mu_{i} \Longrightarrow \frac{\mathrm{d} y}{\mathrm{~d} x}=-\frac{\mu_{i}}{1-\mu_{r}}
\end{aligned}
$$

where the solutions of these equations are the curves $u, v=$ constant. The lens equation is therefore uniquely determined if the values are known on curves (not identical to characteristics). Even without this knowledge, characteristics are of interest: each two (possibly) multiply-intersecting characteristics of $u$ and $v$ map onto a cross-hair in the source plane so that multiply imaged arclets can be identified.

\section{REFERENCES}

Schramm, T. \& Kayser, R. 1995, A\&A, 299, 1 